\title{
Passt der Beruf zu mir?
}

\section{Determinanten und Konsequenzen wahrgenommener Passung mit dem Lehrberuf beim Übergang in die Berufsbildung}

\author{
Christof Nägele \& Markus P. Neuenschwander
}

\section{Zusammenfassung}

Beim Übergang von der Schule in die berufliche Grundbildung müssen sich die Jugendlichen einer sich verändernden sozialen Situation anpassen. Dies gelingt ihnen besser, wenn sie einen Lehrberuf gewählt haben, den sie als passend zu ihren Interessen und Fähigkeiten wahrnehmen. Die wahrgenommene Passung ist ein Ergebnis des Berufswahlprozesses und kann sich zu Beginn der beruflichen Grundbildung aufgrund neuer Erfahrungen verändern. Die Ergebnisse von Pfadanalysen zeigen, dass wichtige Prädiktoren der wahrgenommenen Passung mit dem Lehrberuf zu Beginn der berulichen Grundbildung, die wahrgenommene Passung mit dem Lehrberuf, die Entscheidungssicherheit und die Absicht, die Lehre abzuschliessen, am Ende des 9. Schuljahres sind. Die wahrgenommen Passung während der Lehre ist zudem ein guter Prädiktor distaler Ergebnisse der betrieblichen Sozialisation (Zufriedenheit mit der Lehre und dem Ausbildungsbetrieb, Einschätzung des Lernfortschritts und Absicht, die Lehre abzuschliessen). Die Ergebnisse zeigen, dass die Wahl des Ausbildungsbetriebs sowie die Einführungs- und Sozialisationsstrategien der Betriebe mehr Aufmerksamkeit erhalten sollten. 


\section{Abstract}

In transition from school to initial vocational education and training (iVET) adolescents' need to adapt to a changing social situation. This transition will be smoother if the adolescents have chosen an occupation that they perceive as well fitting to their personal interests and abilities. The perceived person-iVET fit is a result of the vocational choice process. This fit is assumed to change based on new experiences during transition to work. Path analyses show that important predictors of the person-iVET fit in the beginning of iVET are the perceived person-iVET fit, the confidence in the vocational choice and the intention to finish iVET, measured at the end of compulsory school. Furthermore, we can show that the perceived fit during iVET is a predictor of distal outcomes of the organizational socialization processes (work satisfaction, occupational satisfaction, assessment of the own progress in learning, intention to finish iVET). The results show that the choice of the training company as well as the insertion of the new apprentices in the training companies should get more attention.

\section{Résumé}

Lors du passage de l'école dans la formation professionnelle initiale, il faut que les jeunes s'adaptent à une situation sociale changeante. Ils y parviennent mieux s'ils ont choisi une profession qu'ils perçoivent comme conforme à leurs intérêts et leurs capacités. La conformité perçue est le résultat du processus de l'orientation professionnelle et peut changer au début de la formation professionnelle initiale à cause des expériences nouvelles. Les résultats des analyses des pistes causales montrent que des prédicteurs importants de la conformité perçue avec la profession, au début de la formation professionnelle initiale, sont la conformité perçue avec la profession, la sécurité décisionnelle et l'intention de terminer l'apprentissage, mesure à la fin de leécole obligatoire. De plus, la conformité perçue pendant l'apprentissage est un bon prédicteur des résultats distaux de la socialisation en entreprise (la satisfaction avec l'apprentissage et l'entreprise formatrice, l'estimation des progrès d'acquis et l'intention de terminer l'apprentissage). Les résultats montrent qu'il faudrait porter plus d'attention au choix de l'entreprise formatrice ainsi qu'aux stratégies d'introduction et de socialisation des entreprises. 


\section{Wahrgenommene Passung beim Übergang in die Berufsbildung}

Ein wichtiges Ergebnis des Berufswahlprozesses ist, dass die Schülerinnen und Schüler einen Lehrberuf gewählt haben, den sie als zu ihren Interessen, Fähigkeiten und Neigungen passend wahrnehmen. Beim Übergang in die berufliche Grundbildung (Lehrberuf) wird diese wahrgenommene Passung mit dem Lehrberuf aufgrund neuer Erfahrungen angepasst. Die wahrgenommene Passung mit dem Lehrberuf ist folglich auch ein wichtiges Ergebnis der betrieblichen Sozialisation und ein Indikator für eine erfolgreiche Anpassung. Eine erfolgreiche Anpassung und damit eine hohe wahrgenommene Passung führen zu einer hohen Zufriedenheit mit dem Lehrberuf und dem Lehrbetrieb, einer hohen Absicht, die Lehre abzuschliessen und einer positiven Einschätzung des eigenen Lernfortschritts (Kammeyer-Mueller \& Wanberg, 2003). Vermittelt über soziale Prozesse führt die wahrgenommene Passung auch zu einem hohen beruflichen Commitment und zu einem hohen betrieblichen Commitment (Nägele \& Neuenschwander, 2014).

Dieses Kapitel trägt zur Diskussion der betrieblichen Sozialisation von neu in eine Organisation eintretenden Personen beim Übergang von der Schule in Arbeit und Beruf bei und gibt Hinweise zur Optimierung des Übergangs von der Schule in die berufliche Grundbildung mit Blick auf den Berufswahlprozess und die Einführung von Lernenden in den Ausbildungsbetrieben. Es wird zuerst der Frage nachgegangen, wie sich die wahrgenommene Passung mit dem Lehrberuf im 9. Schuljahr und in den ersten fünf Monaten der beruflichen Grundbildung verändert. Es wird dann die Frage gestellt, welchen Einfluss Ergebnisse des Berufswahlprozesses auf die wahrgenommene Passung im ersten Monat der beruflichen Grundbildung haben. Schliesslich wird die Frage bearbeitet, welchen Einfluss die wahrgenommene Passung mit dem Lehrberuf im fünften Monat der beruflichen Grundbildung auf die distalen Ergebnisse betrieblicher Sozialisation (die Zufriedenheit mit der Lehre und dem Ausbildungsbetrieb, die Einschätzung des Lernfortschritts und die Absicht, die Lehre abzuschliessen) hat.

\subsection{Wahrgenommene Passung}

Passung wird definiert als die Übereinstimmung eines Individuums mit seinem Ausbildungs- und Arbeitsumfeld, wenn Eigenschaften der Person und seiner Umwelt gut zusammenpassen (Kristof, 1996). Das Arbeitsumfeld kann die Tätigkeit, die Arbeit, den Beruf, den Betrieb, die Arbeitsgruppe oder andere Aspekte umfas- 
sen (Kristof-Brown, Zimmerman, \& Johnson, 2005). Die Passung kann anhand objektiver Kriterien festgelegt sein (z.B. Qualifikationserfordernisse der Arbeit und Qualifikation der Person) oder sie kann auf einer subjektiven Wahrnehmung der Situation beruhen.

Die Frage nach der wahrgenommenen Passung wird in Zusammenhang mit der Berufswahl (Bergmann, 2007; Holland, 1959), der Personalselektion (Görlich \& Schuler, 2007; Lengnick-Hall, Lengnick-Hall, Andrade, \& Drake, 2009), und den betrieblichen Einführungs- und Sozialisationsprozessen diskutiert (Kammeyer-Mueller \& Wanberg, 2003). Die Wichtigkeit der wahrgenommenen Passung begründet sich generell in frühen Theorien zur Berufswahl von Holland (1959), in aktuellen, konstruktivistischen Berufswahl- und Laufbahntheorien (Hirschi, 2013) und auch darin, dass ein Beruf nur dann ein positiver und sinnhafter Ausdruck der eigenen Person sein kann, wenn dieser zu den eigenen Interessen und Fähigkeiten passt.

Eine hohe Passung von Person und Beruf führt zu einer höheren beruflichen Zufriedenheit und Einschätzung des beruflichen Erfolgs, die sich auch in einem besseren Verlauf der Karriere zeigt (Hirschi, Niles, \& Akos, 2011; Holland, 1959; Neuenschwander, Gerber, Frank, \& Rottermann, 2012). Die Frage nach der wahrgenommenen Passung ist auch wichtig, da eine geringe wahrgenommene Passung mit der gewählten Lehre und damit dem Beruf, eher dazu führt, dass Probleme in der beruflichen Grundbildung entstehen, die zu einer sinkenden Motivation, zu Konflikten oder zu Lehrvertragsauflösungen führen (Neuenschwander \& Nägele, 2014). Jugendliche mit einer hohen wahrgenommenen Passung haben ein geringeres Risiko nach Abschluss der beruflichen Grundbildung arbeitslos zu werden oder den Beruf zu wechseln (Neuenschwander et al., 2012). Personen mit einer hohen antizipierten Passung mit der zukünftigen Arbeit, dem Betrieb und Beruf fällt der Einstieg und die Anpassung in der neuen Arbeit leichter, als Personen mit einer tiefen wahrgenommenen Passung (Cable \& Judge, 1996). Diese Personen verbleiben eher in einer Organisation, sind leichter zu motivieren und erbringen bessere Leistungen (Kristof, 1996; Taris, Feij, \& Capel, 2006). Eine hohe wahrgenommene Passung von Person und Beruf wird als eine Voraussetzung zur Entwicklung einer hohen wahrgenommenen Passung von Person und Betrieb gesehen (Vogel \& Feldman, 2009) und ist deren Entwicklung vorgelagert. Diese Aussage ist bedeutsam für die Wahl einer beruflichen Grundbildung, da im Berufswahlprozess in aller Regel zuerst der Lehrberuf gewählt wird und erst in einem nachfolgenden Schritt der Ausbildungsbetrieb.

In diesem Beitrag steht die wahrgenommene Passung der Jugendlichen im Sinne einer wahrgenommenen Ähnlichkeit mit der gewählten beruflichen Grundbildung (Lehre) und damit dem gewählten Lehrberuf im Vordergrund. Mit der 
wahrgenommenen Passung mit dem Lehrberuf bezeichnen wir eine subjektive, selbst eingeschätzte Übereinstimmung der eigenen Interessen und Fähigkeiten mit der gewählten, respektive realisierten Lehre. Die wahrgenommene Passung entwickelt sich aufgrund der subjektiven Wahrnehmung der Situation, individueller Vergleichsprozesse und der Interaktion der Person mit der Situation.

\section{2 Übergang von der Schule in die berufliche Grundbildung}

Frühere Studien zeigen, dass sich bei Erwachsenen nach Antritt einer neuen Stelle die wahrgenommene Passung verändert (Kammeyer-Mueller \& Wanberg, 2003). Eine Veränderung der wahrgenommenen Passung erfolgt aufgrund neuer Erfahrungen, die durch den Verlauf der betrieblichen Sozialisation beeinflusst sind. Mit der betrieblichen Sozialisation sind Anpassungsprozesse an die neue soziale Situation angesprochen, die auf den Bemühungen der Lernenden oder des Lernenden beruhen, sich in den Ausbildungsbetrieb zu integrieren und auch den Bemühungen des Ausbildungsbetriebs, die Lernenden zu integrieren.

Der Berufswahlprozess und der Übergang in die berufliche Grundbildung umfassen mehrere Phasen, so dass die wahrgenommene Passung mit dem Lehrberuf auf unterschiedlichen Erfahrungen und Aktivitäten beruht, die auch Bewerbungs- und Selektionsverfahren umfassen (Herzog, Neuenschwander, \& Wannack, 2001, 2006). Die wahrgenommene Passung mit dem Lehrberuf dürfte daher auf vielfältigen und realistischen Informationen über den Lehrberuf und den Ausbildungsbetrieb basieren. Je realistischer Informationen über den zukünftigen Beruf und Betrieb sind, desto leichter fällt die Anpassung in den ersten Monaten in der neuen Organisation (Werbel, Landau, \& DeCarlo, 1996). Realistische Information heisst, dass sowohl die positiven als auch die negativen Aspekte eines Berufs oder Betriebs bekannt sind und in die Berufswahl einfliessen. Dieser realistische Blick auf den zukünftigen Beruf ist vielen Jugendlichen möglich, da sie umfassende Informationen verfügbar haben und in der Regel auch Schnupperlehren absolvieren. Diese Ausganslage und Ergebnisse früherer Studien zum Übergang von der Schule in eine Ausbildung auf Sekundarstufe II (Neuenschwander, 2007) lassen deshalb vermuten, dass sich die im Berufswahlprozess erarbeitete wahrgenommene Passung mit dem Lehrberuf beim Eintritt in die berufliche Grundbildung unmittelbar nur wenig verändern dürfte. Es konnte gezeigt werden, dass ein starker Rückgang der wahrgenommenen Passung vor allem dann auftritt, wenn wenig Wissen über den zukünftigen Beruf und den Betrieb vorliegt oder die Erwartungen vor dem Eintritt in die Organisation un- 
realistisch hoch waren (Jones, 1986; Taris et al., 2006). Obwohl die Entscheidung für eine berufliche Grundbildung auf unvollständigen Informationen beruht (Neuenschwander \& Hermann, 2014), haben die Jugendlichen im Berufswahlprozess in der Regel eine gute Vorstellung über den zukünftigen Beruf erworben. Der Wahl der beruflichen Grundbildung geht in der Regel eine intensive Phase der Berufserkundung und Berufsfindung voran, weshalb wir annehmen, dass die Schülerinnen und Schüler unmittelbar vor Beginn der beruflichen Grundbildung viel Wissen über den Beruf haben und ihre Erwartungen aufgrund der Selektionsmechanismen nicht überhöht sind.

Hypothese 1: Die Stärke der wahrgenommenen Passung der Jugendlichen mit dem Lehrberuf wird sich beim direkten Übergang von der Schule in die berufliche Grundbildung im ersten Monat nicht verändern.

Für die berufliche und betriebliche Sozialisation stellen der Beginn der beruflichen Grundbildung und die ersten Monate in der Ausbildung wichtige Phasen dar. Allfällige Probleme, die später zu Konflikten und Lehrabbrüchen führen können, beginnen sich bereits in dieser frühen Phase abzuzeichnen (Berweger, Krattenmacher, Salzmann, \& Schönenberger, 2013; Schmid, 2010). Die Stärke der wahrgenommenen Passung mit dem Lehrberuf verändert sich abhängig von den Erfahrungen und den betrieblichen Sozialisationsprozessen (Kammeyer-Mueller, Wanberg, Rubenstein, \& Song, 2013). Es kann deshalb vermutet werden, dass es unterschiedliche Entwicklungsverläufe der wahrgenommenen Passung mit dem Lehrberuf gibt. Die wahrgenommene Passung mit dem Lehrberuf kann beim Übergang von der Schule in den Lehrbetrieb steigen oder sinken. Sie kann zudem auch im weiteren Verlauf der beruflichen Grundbildung steigen oder sinken.

Hypothese 2: Es können unterschiedliche Entwicklungsverläufe der wahrgenommenen Passung mit dem Lehrberuf identifiziert und beschrieben werden.

\subsection{Prädiktoren der wahrgenommenen Passung mit dem Lehrberuf im ersten Monat der beruflichen Grundbildung}

Die im Berufswahlprozess erarbeitete wahrgenommene Passung mit dem Lehrberuf ist ein wichtiger Prädiktor der wahrgenommenen Passung mit dem Lehrberuf im ersten Monat der beruflichen Grundbildung (Neuenschwander et al., 2012). Darüber hinaus ist die wahrgenommene Passung mit dem Lehrberuf im 
ersten Monat der beruflichen Grundbildung von weiteren Faktoren abhängig: Entscheidungssicherheit, den richtigen Beruf und den richtigen Ausbildungsbetrieb gewählt zu haben, und die Zufriedenheit mit der Berufswahl und der Absicht, die Lehre abzuschliessen.

Die Entscheidung für eine berufliche Grundbildung wird durch rationale und situative Prozesse gesteuert und basiert auf den jeweils verfügbaren Informationen, emotionalen Prozessen, der Rückmeldung relevanter Bezugspersonen, eigenen Erfahrung im Berufswahlprozess und der Bewerbungsphase für eine Lehrstelle (Neuenschwander \& Hermann, 2014). Daraus resultiert die Einstellung, den richtigen Beruf und den richtigen Lehrbetrieb gewählt zu haben. Diese individuelle Entscheidungssicherheit hat einen positiven Einfluss auf die wahrgenommene Passung mit dem Lehrberuf (Singer, Gerber, \& Neuenschwander, 2014). Grundsätzlich beeinflusst die wahrgenommene Passung die Bewertung der Arbeit, so auch die Zufriedenheit (Cable \& Judge, 1996). Die Zufriedenheit mit der Arbeit ist ein wichtiger Indikator des beruflichen Erfolgs (Abele-Brehm, 2014; Kammeyer-Mueller \& Wanberg, 2003; Keller, Semmer, Samuel, \& Bergman, 2014), der aufgrund einer subjektiven, kognitiven Bewertung der Situation entsteht. Die Zufriedenheit mit der gewählten Lehre ist so ein Indikator für einen positiv verlaufenen Berufswahlprozess. Wir vermuten deshalb, dass die Zufriedenheit mit der Berufswahl, die wahrgenommene Passung mit dem Lehrberuf im ersten Monat der beruflichen Grundbildung vorhersagt. Die Absicht, die Lehrstelle zu wechseln, und der tatsächliche Lehrstellenwechsel sind von grosser Bedeutung für die Lernenden und für die Betriebe. Die Gründe, eine Lehre nicht abschliessen zu wollen, sind vielfältig (Keller \& Stalder, 2012; Neuenschwander, 1997). Personen, die jedoch Zweifel hegen, ob sie ihre berufliche Grundbildung abschliessen werden, schauen sich eher nach Alternativen um und engagieren sich oft weniger in der Ausbildung und Arbeit. Die Absicht, etwas tun zu wollen, ist ein unmittelbarer Vorläufer der tatsächlichen Ausführung eines bestimmten Verhaltens (Ajzen, 2002). Die Herausbildung einer Absicht hängt von situativen Faktoren (Beanspruchung, Belastung, Zufriedenheit) und individuellen Faktoren ab (Kontrolle, Selbstwert, soziale Unterstützung) (Firth, Mellor, Moore, \& Loquet, 2004). Die Absicht, die Lehre abzuschliessen, dürfte deshalb die wahrgenommen Passung mit dem Lehrberuf beeinflussen.

Hypothese 3: a) Die wahrgenommene Passung mit dem Lehrberuf, b) die Entscheidungssicherheit, den richtigen Beruf gewählt zu haben, c) die Entscheidungssicherheit, den richtigen Lehrbetrieb gewählt zu haben, d) die Zufriedenheit mit der Lehre und e) die Absicht, die Lehre abzuschliessen, - gemessen jeweils am 
Ende des 9. Schuljahres - sagen die wahrgenommene Passung mit dem Lehrberuf im ersten Monat der beruflichen Grundbildung vorher.

\subsection{Wahrgenommene Passung als Prädiktor distaler Ergebnisse betrieblicher Sozialisation}

Die wahrgenommene Passung mit dem Lehrberuf verändert sich nach Beginn der beruflichen Grundbildung. Aktuelle Studien zeigen, dass sich vor allem Arbeitsinhalte und die Möglichkeit der selbständigen Regulierbarkeit der eigenen Tätigkeit positiv auf die wahrgenommene Passung auswirken (Neuenschwander, 2011). Die Veränderung der wahrgenommenen Passung mit dem Lehrberuf während der beruflichen Grundbildung scheint über Prozesse der sozialen Integration und Aufgabenbeherrschung vermittelt zu sein (Neuenschwander, 2011; Singer et al., 2014). Wir gehen jedoch von einer insgesamt hohen Stabilität der wahrgenommenen Passung in den ersten Monaten der beruflichen Grundbildung aus.

Hypothese 4: Wir nehmen an, dass die wahrgenommene Passung mit dem Lehrberuf zu Beginn der beruflichen Grundbildung die Passung mit dem Lehrberuf im fünften Monat in der beruflichen Grundbildung vorhersagt.

Basierend auf einem Modell der betrieblichen Sozialisation von neu in einen Betrieb eintretenden Personen (Kammeyer-Mueller \& Wanberg, 2003), werden die Zufriedenheit mit der Lehre, die Zufriedenheit mit dem Betrieb, der Lernfortschritt und die Absicht, die Lehre abschliessen zu wollen, als distale Ergebnisse der betrieblichen Sozialisation betrachtet. Diese distalen Ergebnisse der betrieblichen Sozialisation sind durch die wahrgenommene Passung mit dem Lehrberuf beeinflusst, da eine hohe wahrgenommene Passung die Anpassung erleichtert (Kristof, 1996; Kristof-Brown et al., 2005).

Die Zufriedenheit mit der Lehre, die Zufriedenheit mit dem Betrieb als subjektive, kognitive Bewertung der Situation, die Einschätzung des eigenen Lernfortschritts und die Absicht, die Lehre abschliessen zu wollen, sind Indikatoren einer erfolgreichen Anpassung der Lernenden und eines positiven Verlaufs der beruflichen Grundbildung. Die Einschätzung des eigenen Lernfortschritts beruht auf den Lernmöglichkeiten im Betrieb (Taris et al., 2006), die von individuellen, betrieblichen und strukturellen Faktoren abhängig sind (Nägele, 2013). Eine positive Wahrnehmung des eigenen Lernfortschritts bedeutet, dass sich die Jugendlichen in der beruflichen Grundbildung entwickeln können. Dies ist deshalb ein 
wichtiges Ergebnis der Anpassungsprozesse zu Beginn der beruflichen Grundbildung.

Hypothese 5: Wir erwarten, dass die wahrgenommene Passung mit dem Lehrberuf im fünften Monat der beruflichen Grundbildung a) die Zufriedenheit mit der Lehre, b) die Zufriedenheit mit dem Betrieb, c) den Lernfortschritt und d) die Absicht, die Lehre abschliessen zu wollen, nach sechs Monaten in der beruflichen Grundbildung vorhersagt.

Wir erwarten, dass die Zufriedenheit mit der Lehre am Ende des 9. Schuljahrs die Zufriedenheit mit der Lehre im sechsten Monat der beruflichen Grundbildung vorhersagt (Hypothese 6a), wie auch die Absicht die Lehre abzuschliessen im 9. Schuljahr die Absicht, die Lehre abzuschliessen im sechsten Monat der beruflichen Grundbildung vorhersagt (Hypothese 6b). Die Hypothesen 3 bis 6 sind im Modell in Abbildung 1 dargestellt.

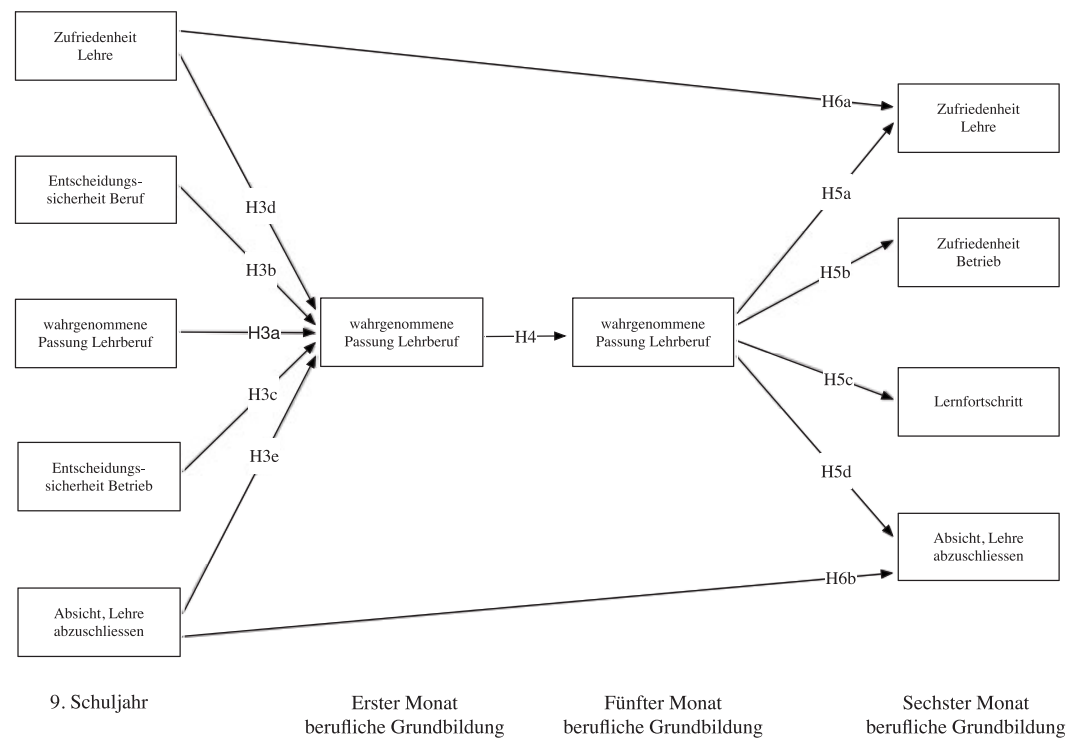

Abbildung 1 Postuliertes Pfadmodell. 


\section{$2 \quad$ Methode}

Die Daten zur Überprüfung des postulierten Pfadmodells (Abbildung 1) stammen aus dem Projekt Sozialisationsprozesse beim Übergang in den Lehrbetrieb (SoLe) (Neuenschwander \& Nägele, 2014). Diese Studie wurde finanziell unterstützt durch das Staatssekretariat für Bildung, Forschung und Innovation (SBFI). Es handelt sich um eine Längsschnittstudie mit sieben Messzeitpunkten. Die Jugendlichen wurden erstmalig am Ende des 9. Schuljahres und dann monatlich nach Beginn der beruflichen Grundbildung mittels Online-Fragebogen befragt. Es wurde im 9. Schuljahr eine repräsentative Stichprobe von Jugendlichen ausgewählt und für die Teilnahme angefragt. Es wurden Jugendliche ausgewählt, die ihre berufliche Grundbildung direkt im Anschluss an die obligatorische Schule begannen. Die Befragungen fanden von April 2011 bis Januar 2012 in verschiedenen Kantonen in der Deutschschweiz statt (Neuenschwander, Gerber, Frank, \& Bosshard, 2013). Die erste Befragung erfolgte im 9. Schuljahr im Klassenverband mit einem Online-Fragebogen. Während der beruflichen Grundbildung wurden die Lernenden individuell per Email zum Ausfüllen des Online-Fragebogens eingeladen.

\subsection{Stichprobe}

Es wurden in den Kantonen Aargau, Bern, Luzern, Obwalden Schaffhausen, Schwyz, St. Gallen und Thurgau proportional zur Kantonsgrösse 220 Schulen für die Teilnahme an der Studie angefragt. 67 Schulen (31\%) erklärten sich zur Teilnahme bereit. Die Ergebnisse der hier präsentierten Analysen beruhen auf Angaben von Jugendlichen, die den Fragebogen im 9. Schuljahr ausgefüllt hatten, direkt im Anschluss an die Schule eine berufliche Grundbildung begannen und sich an den monatlichen Befragungen während der ersten sechs Monate der beruflichen Grundbildung beteiligten. Die Analysen beruhen auf Daten aus vier Messzeitpunkten (9. Schuljahr $N=505$, erster Monat in der beruflichen Grundbildung $N=453$, fünfter Monat in der beruflichen Grundbildung $N=413$, sechster Monat in der beruflichen Grundbildung $N=412$ ). Diese Monate wurden gewählt, um den unmittelbaren Übertritt von der Schule in die berufliche Grundbildung und die Ergebnisse des Anpassungsprozesses nach der Einführungsphase darzustellen. Es wurden keine systematischen Rücklaufverzerrungen zwischen den einzelnen Erhebungszeitpunkten gefunden (Neuenschwander \& Nägele, 2014). $23 \%$ der Schülerinnen und Schüler stammten aus Schulniveaus mit Grundanfor- 
derungen, 51\% waren Schülerinnen und der Altersdurchschnitt lag am Ende des 9. Schuljahrs bei 15.7 Jahren $(S=.56, N=501)$.

\subsection{Instrumente}

Die wahrgenommene Passung der Jugendlichen mit dem Lehrberuf wurde mit drei Items gemessen (Neuenschwander \& Frank, 2009): „Meine Lehre passt zu meiner Person“, „Meine Lehre stimmt mit meinen persönlichen Interessen überein" und „Meine Lehre stimmt mit meinen beruflichen Fähigkeiten überein“, auf einer Antwortskale von 1 „stimmt überhaupt nicht“ bis 6 "stimmt voll und ganz". Die interne Konsistenz der Skala war im 9. Schuljahr Cronbach's $\alpha=.80$, im ersten Monat der beruflichen Grundbildung $\alpha=.88$ und im fünften Monat $\alpha=.90$.

Die Entscheidungssicherheit, sich für den richtigen Beruf richtige Lehre und damit den richtigen Beruf entschieden zu haben, wurde im 9. Schuljahr mit drei Items gemessen (Neuenschwander et al., 2013): „Ich bin mir sicher, dass meine Berufsentscheidung die richtige für mich ist“, „Ich bin manchmal verunsichert, ob ich mich für den richtigen Beruf entschieden habe“ (rekodiert), und „Ich stehe voll und ganz zu meiner Berufswahl“ auf einer Antwortskala von 1 „stimmt überhaupt nicht" bis 6 „stimmt voll und ganz“. Die interne Konsistenz der Skala war Cronbach's $\alpha=.86$.

Die Entscheidungssicherheit, sich für den richtigen Lehrbetrieb entschieden zu haben, wurde im 9. Schuljahr mit drei Items gemessen (Neuenschwander et al., 2013): „Wenn ich die Wahl zwischen verschiedenen Lehrbetrieben hätte, würde ich mich dennoch für meinen zukünftigen Lehrbetrieb entscheiden“, „Ich freue mich, gerade in diesem Lehrbetrieb meine Berufsausbildung zu machen" und „Ich bin manchmal unsicher, ob ich meine zukünftige Lehre im richtigen Lehrbetrieb machen werde“ (rekodiert), auf einer Antwortskala von 1 „stimmt überhaupt nicht“ bis 6 ,stimmt voll und ganz“. Die interne Konsistenz der Skala war Cronbach's $\alpha=.80$.

Die Zufriedenheit mit der Lehre wurde im 9. Schuljahr und im sechsten Monat der beruflichen Grundbildung mit einem Item gemessen (Neuenschwander et al., 2013): „Wie zufrieden bist du zum jetzigen Zeitpunkt mit deiner Berufslehre?“, auf einer Antwortskala von 1 „überhaupt nicht zufrieden“ bis 6 „,voll und ganz zufrieden“. Im 9. Schuljahr war die Frage, wie zufrieden die Schülerin oder der Schüler mit der zukünftigen Berufslehre sei.

Die Zufriedenheit mit dem Lehrbetrieb wurde im sechsten Monat der beruflichen Grundbildung mit einem Item gemessen (Neuenschwander et al., 2013): 
„Wie zufrieden bist du zum jetzigen Zeitpunkt mit deinem Lehrbetrieb?“ auf einer Antwortskale von 1 ,überhaupt nicht zufrieden“ bis 6 ,voll und ganz zufrieden“.

Die Absicht, die Lehre abzuschliessen, wurde mit drei Items gemessen (Neuenschwander et al., 2013): „Ich will meine Lehre unbedingt abschliessen“, „Ich kann mir vorstellen, meine Lehre abzubrechen“ (rekodiert) und „Nichts ist mir wichtiger, als den Lehrabschluss zu machen“, auf einer Antwortskala von 1 „stimmt überhaupt nicht“ bis 5 ,stimmt voll und ganz“. Die interne Konsistenz der Skala war im 9. Schuljahr Cronbach's $\alpha=.55$, im sechsten Monat der beruflichen Grundbildung $\alpha=.80$.

Der selbst eingeschätzte Lernfortschritt wurde im sechsten Monat der beruflichen Grundbildung mit einem Item gemessen (Neuenschwander et al., 2013): „Wie viel hast du seit deinem Lehrbeginn gelernt?", auf einer Antwortskala von 1 „überhaupt nicht viel“ bis 6 „sehr viel“.

\section{$3 \quad$ Ergebnisse}

Vor der Datenanalyse wurden die Daten auf Extremwerte (extrem hohe und tiefe Werte) und die Einhaltung der Normalverteilung überprüft und entsprechend der Empfehlung von Tabachnick und Fidell (2007) korrigiert. Diese Massnahmen erhöhen die Zuverlässigkeit der Ergebnisse, da diese so weniger von seltenen und extremen Ausprägungen in den Daten verfälscht werden.

\subsection{Deskriptive Ergebnisse}

In Tabelle 1 sind die Mittelwerte und Standardabweichungen der Entscheidungssicherheit für den Beruf und die Entscheidungssicherheit für den Betrieb am Ende des 9. Schuljahres und der distalen Ergebnisse der betrieblichen Sozialisation (Zufriedenheit mit der Lehre, Zufriedenheit mit dem Betrieb, Lernfortschritt und die Absicht, die Lehre abzuschliessen) dokumentiert. 
Tabelle 1 Anzahl Jugendliche, Mittelwerte und Standardabweichungen aller Variablen im Modell.

\begin{tabular}{lccc}
\hline & Anzahl & Mittelwert & $\begin{array}{c}\text { Standard- } \\
\text { abweichung }\end{array}$ \\
\hline 9. Schuljahr & & & \\
\hline Entscheidungssicherheit Beruf & 499 & 5.67 & .29 \\
\hline Entscheidungssicherheit Betrieb & 503 & 5.70 & .28 \\
\hline Zufriedenheit mit der Lehre & 505 & 5.90 & .17 \\
\hline Absicht die Lehre abzuschliessen & 503 & 5.90 & .14 \\
\hline Sechster Monat berufliche Grundbildung & & & \\
\hline Zufriedenheit mit der Lehre & 406 & 5.71 & .25 \\
\hline Lernfortschritt & 406 & 5.73 & .30 \\
\hline Zufriedenheit mit dem Lehrbetrieb & 397 & 5.68 & .28 \\
\hline Absicht die Lehre abzuschliessen & 409 & 5.79 & .20 \\
\hline
\end{tabular}

\subsection{Veränderung der wahrgenommenen Passung mit dem Lehrberuf}

Die wahrgenommene Passung mit dem Lehrberuf war bei den direkt nach der Schule mit einer beruflichen Grundbildung beginnenden Jugendlichen hoch (Tabelle 2). Die wahrgenommene Passung mit dem Lehrberuf stieg beim Übergang von der Schule in den Lehrbetrieb weiter an und sank bis im fünften Monat der beruflichen Grundbildung wieder leicht. Eine Varianzanalyse mit Messwiederholung zeigte, dass dieser Anstieg und das anschliessend Sinken der wahrgenommenen Passung mit dem Lehrberuf statistisch signifikant waren, $F(2,744)=1.72, p<$ $.01, \eta^{2}=.10$. Kontrastanalysen zeigten, dass sowohl die wahrgenommene Passung mit dem Lehrberuf im ersten Monat $F(1,372)=67.27, p<.01, \eta^{2}=.15$ und im fünften Monat $F(1,372)=31.61, p<.01, \eta^{2}=.08$ höher waren als die wahrgenommene Passung mit dem Lehrberuf im 9. Schuljahr. Das Sinken der wahrgenommenen Passung vom ersten Monat bis zum fünften Monat in der beruflichen Grundbildung war ebenfalls statistisch signifikant $F(1,372)=8.80, p<.05, \eta^{2}=.02$. Hypothese 1 kann somit nicht bestätigt werden. Denn die wahrgenommene Passung mit dem Lehrberuf stieg beim Übergang von der Schule in die berufliche Grundbildung insgesamt statistisch signifikant an. 
Tabelle 2 Wahrgenommene Passung mit dem Lehrberuf: Anzahl Jugendliche, Mittelwerte und Standardabweichungen

\begin{tabular}{lccc}
\hline & Anzahl & Mittelwert & $\begin{array}{c}\text { Standardabwei- } \\
\text { chung }\end{array}$ \\
\hline 9. Schuljahr & 504 & 5.63 & .33 \\
\hline Berufliche Grundbildung & & & .23 \\
\hline Erster Monat & 451 & 5.75 & .25 \\
\hline Fünfter Monat & 398 & 5.71 & \\
\hline
\end{tabular}

Zur Beschreibung des Verlaufs und der Veränderung der wahrgenommenen Passung mit dem Lehrberuf (9. Schuljahr, erster und fünfter Monat der beruflichen Grundbildung) wurden Verläufe a-priori festgelegt. Die wahrgenommene Passung mit dem Lehrberuf kann ausgehend von einer hohen oder tiefen Ausprägung im 9. Schuljahr zwischen den einzelnen Zeitpunkten gleich bleiben/steigen oder sinken. Die Kategorie gleich/steigend wird als steigend bezeichnet, da sich die wahrgenommene Passung bei allen beobachteten Jugendlichen in dieser Kategorie zwischen den Zeitpunkten erhöhte. So können acht Verläufe beschrieben werden (Abbildung 2). Mittels einer Konfigurationsfrequenzanalyse wurde anschliessend geprüft, welche dieser Verläufe Typen oder Anti-Typen darstellen (Krauth, 1993; von Eye, 2002). Typen sind Verläufe, die häufiger als erwartet beobachtet werden. Anti-Typen sind Verläufe, die seltener als erwartet beobachtet werden. Die Veränderung der wahrgenommenen Passung mit dem Lehrberuf wurde berechnet, indem die wahrgenommene Passung mit dem Lehrberuf im ersten Monat der beruflichen Grundbildung am Mittelwert und der Standardabweichung der wahrgenommenen Passung mit dem Lehrberuf im 9. Schuljahr standardisiert wurde. Werte, die grösser als 0 sind, drücken damit eine Zunahme der wahrgenommenen Passung mit dem Lehrberuf aus, Werte kleiner als 0 eine Abnahme. Die wahrgenommene Passung mit dem Lehrberuf im fünften Monat der beruflichen Grundbildung wurde entsprechend an der wahrgenommenen Passung mit dem Lehrberuf im ersten Monat der beruflichen Grundbildung standardisiert. Die Ergebnisse sind in Abbildung 2 grafisch dargestellt.

Insgesamt 185 Schülerinnen und Schüler hatten im 9. Schuljahr eine hohe wahrgenommene Passung. Bei 111 stieg die wahrgenommene Passung im ersten Monat und im fünften Monat der beruflichen Grundbildung $\left(\mathrm{N}_{\text {erwartet }}=94.46, \chi^{2}\right.$ $=2.90$, n.s.). Bei 41 stieg die wahrgenommene Passung im ersten Monat und sank im fünften Monat wieder $\left(\mathrm{N}_{\text {erwartet }}=35.29, \chi^{2}=.92\right.$, n.s.). Bei 7 sank die wahrgenommene Passung im ersten Monat und stieg im fünften Monat $\left(\mathrm{N}_{\text {erwartet }}=40.22\right.$, 
$\chi^{2}=27.44, p<.01$, Anti-Typ). Bei 26 sank die wahrgenommene Passung im ersten Monat und sank auch im fünften Monat $\left(\mathrm{N}_{\text {erwartet }}=15.03, \chi^{2}=8.01, p<.01\right.$, Typ).

Insgesamt 190 Schülerinnen und Schüler hatten im 9. Schuljahr eine tiefe wahrgenommene Passung. Bei 31 sank die wahrgenommene Passung im ersten Monat und sank auch im fünften Monat der beruflichen Grundbildung $\left(\mathrm{N}_{\text {erwartet }}\right.$ $=15.44, \chi^{2}=15.70, p<.01$, Typ). Bei 48 sank die wahrgenommene Passung im ersten Monat und stieg im fünften Monat wieder $\left(\mathrm{N}_{\text {erwartet }}=41.31, \chi^{2}=1.08\right.$, n.s.). Bei 4 stieg die wahrgenommene Passung im ersten Monat und sank im 5. Monat $\left(\mathrm{N}_{\text {erwartet }}=36.24, \chi^{2}=25.69, p<.01\right.$, Anti-Typ $)$. Bei 107 stieg die wahrgenommene Passung im ersten Monat und stieg auch im fünften Monat $\left(\mathrm{N}_{\text {erwartet }}=97.01, \chi^{2}=\right.$ 1.03 , n.s.).

Das Ergebnis der Konfigurationsfrequenzanalyse in Abbildung 2 zeigt, dass die positiven Verläufe erwartungskonform am häufigsten auftraten (218 Jugendliche, 58\%). Dies war der Verlauf „hoch, dann steigend und steigend“ (111 Jugendliche) und der Verlauf „tief, steigend, steigend“ (107 Jugendliche). Dies widerspiegelt den Anstieg der wahrgenommenen Passung mit dem Lehrberuf beim Übergang von der Schule in die berufliche Grundbildung. Auffallend sind erstens zwei über erwarten häufig auftretende Verläufe. Dies war der Typ „hoch, sinkend, sinkend“ (26 Jugendliche, erwartet 15.44) und der Typ „tief, sinkend, sinkend (31 Jugendlichen, erwartet 15.44). Jugendliche, bei denen die Passung beim Übergang von der Schule in die berufliche Grundbildung zu sinken begann, hatten unabhängig vom Ausgangsniveau der Passung im 9. Schuljahr ein höheres Risiko, dass die Passung bis im fünften Monat der beruflichen Grundbildung weiter sank. Auffallend sind zweitens zwei Verläufe, die seltener auftraten als erwartet. Dies waren die AntiTypen „hoch, sinkend, steigend“ (7 Jugendliche, erwartet 40.22) und der AntiTyp „tief, steigend, sinkend“ (4 Jugendliche, erwartet 36.24). Es war eher unwahrscheinlich, dass Jugendliche mit einer hohen wahrgenommenen Passung mit dem Lehrberuf im 9. Schuljahr, die beim Übergang sank, im weiteren Verlauf wieder eine steigende wahrgenommene Passung mit dem Lehrberuf hatten. Es zeigte sich auch, dass bei Jugendlichen mit einer tiefen wahrgenommenen Passung mit dem Lehrberuf im 9. Schuljahr, die beim Übergang anstieg, diese bis im fünften Monat der beruflichen Grundbildung eher nicht wieder sank. 

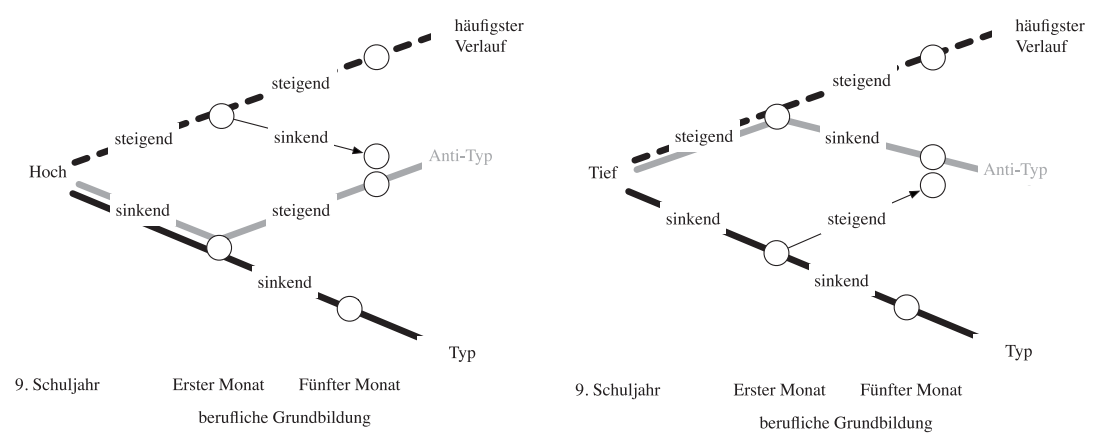

Abbildung 2 Wahrgenommene Passung mit dem Lehrberuf: Verlaufstypen als Ergebnisse der Konfigurationsfrequenzanalyse.

Die wahrgenommene Passung mit dem Lehrberuf veränderte sich bei allen Jugendlichen vom 9. Schuljahr sowohl beim Übergang in die berufliche Grundbildung als auch in deren weiteren Verlauf. Die wahrgenommene Passung mit dem Lehrberuf nahm insgesamt zu. Es gab jedoch Jugendliche, bei denen die wahrgenommene Passung mit dem Lehrberuf beim Übergang von der Schule in die berufliche Grundbildung abnahm. Sank die wahrgenommene Passung mit dem Lehrberuf beim Übergang, bestand ein höheres Risiko, dass diese auch bis zum fünften Monat der beruflichen Grundbildung weiter sank. Jugendliche mit einer tiefen wahrgenommenen Passung mit dem Lehrberuf, die beim Übergang stieg, hatten hingegen eine höhere Chance, dass diese wahrgenommene Passung bis im fünften Monat der beruflichen Grundbildung nicht wieder sank.

Hypothese 2 kann somit bestätig werden. Die wahrgenommene Passung mit dem Lehrberuf verändert sich im Verlauf der ersten fünf Monate in der beruflichen Grundbildung. Es konnten steigende und sinkende Verläufe identifiziert werden. Auffallend ist, dass Jugendliche, bei denen die wahrgenommene Passung mit dem Lehrberuf beim Übergang von der Schule in die berufliche Grundbildung zu sinken begann, ein höheres Risiko hatten, dass sich dieser Trend fortsetzte und auch im fünften Monat der beruflichen Grundbildung nicht umkehrte.

\subsection{Pfadmodell}

Um das in Abbildung 1 postulierte Modell zu überprüfen, wurde ein Pfadmodell mittels Mplus berechnet (Muthén \& Muthén, 1998-2012). Ein Pfadmodell wird 
statistisch anhand dessen Fit Indices beurteilt. Ein guter Fit entsteht, wenn die im Modell postulierten Beziehungen (Pfade) anhand der vorliegenden Daten bestätigt werden. Der Fit wird dann als gut bewertet, wenn das Verhältnis von $\chi^{2}$ geteilt durch die Freiheitsgrade (df) kleiner als 5 und grösser als 1 ist (Schumacker \& Lomax, 1996), wenn der comparative fit index (CFI) grösser als .95 ist, der Tucker Lewis index (TLI) grösser als .95, das standardized root Mean square residual (SRMR) kleiner als .08 und die root mean square error of approximation (RMSEA) kleiner als .05 sind (Hu \& Bentler, 1999).

Das in Abbildung 1 postulierte Pfadmodell erzielte einen guten Fit $\chi^{2}(27)=$ $55.82, p<.01, \mathrm{CFI}=.974, \mathrm{TLI}=.956, \mathrm{SRMR}=.070, \mathrm{RMSEA}=.051$. In diesem Modell wurden jedoch nicht alle postulierten Pfade statistisch signifikant. Der Fit dieses Modells konnte weiter verbessert werden, indem zwei Pfade zusätzlich hinzugefügt wurden. Der Fit dieses um zwei Pfade ergänzten Modells war $\chi^{2}(25)=$ $38.91, p<.01$, CFI $=.987$, TLI $=.977$, SRMR $=.065$, RMSEA $=.037, \Delta \chi^{2}(3)=16.91$, $p<.01$. Wir berichten die Pfadkoeffizienten des optimierten Modells gemäss Abbildung 3, inklusive der nicht signifikanten Pfade.

Die wahrgenommene Passung mit dem Lehrberuf am Ende des 9. Schuljahres hatte einen direkten Einfluss auf die wahrgenommene Passung mit dem Lehrberuf im ersten Monat der beruflichen Grundbildung ( $\beta=.38, p<.01$, Hypothese 3a bestätigt). Die wahrgenommene Passung mit dem Lehrberuf im ersten Monat sagte die wahrgenommene Passung mit dem Lehrberuf im fünften Monat vorher $(\beta=.69, p<.01$, Hypothese 4 bestätigt). Die Entscheidungssicherheit, den richtigen Beruf gewählt zu haben, war wie postuliert ein Prädiktor der wahrgenommenen Passung mit dem Lehrberuf im ersten Monat der beruflichen Grundbildung ( $\beta=.18, p<.01$, Hypothese $3 \mathbf{b}$ bestätigt). Nicht bestätigt werden konnte die Hypothese 3c, die einen Zusammenhang zwischen der Entscheidungssicherheit, den richtigen Lehrbetrieb gewählt zu haben, und der wahrgenommenen Passung mit dem Lehrberuf im ersten Monat postulierte ( $\beta=.04$, n.s.). Die Hypothese 3d, dass die Zufriedenheit mit der Lehre $(\beta=.11, p<.05)$ und Hypothese 3e, dass die Absicht, die Lehre abzuschliessen $(\beta=.10, p<.05)$, die wahrgenommene Passung mit dem Lehrberuf im ersten Monat vorhersagen, konnten bestätigt werden. Die wahrgenommene Passung mit dem Lehrberuf im fünften Monat sagte die Zufriedenheit mit der Lehre $(\beta=.54, p<.01$, Hypothese $5 \mathrm{a}$ bestätigt), die Zufriedenheit mit dem Betrieb ( $\beta=.39, p<.01$, Hypothese $5 \mathbf{b}$ bestätigt), den Lernfortschritt ( $\beta$ $=.35, p<.01$, Hypothese $5 \mathrm{c}$ bestätigt) und die Absicht, die Lehre abzuschliessen $(\beta=.28, p<.01$, Hypothese $5 \mathbf{d}$ bestätigt), vorher.

Die Zufriedenheit mit der Lehre im 9. Schuljahr hatte einen direkten, Einfluss auf die Zufriedenheit mit der Lehre im sechsten Monat $(\beta=.10, p<.05)$, Hypothese 6a bestätigt). Die Absicht, die Lehre abzuschliessen im 9. Schuljahr, hatte 
einen direkten Einfluss auf die Absicht, die Lehre abzuschliessen zu im sechsten Monat der beruflichen Grundbildung ( $\beta=.32, p<.01$, Hypothese $6 \mathbf{b}$ bestätigt). Die gegenüber dem postulierten Modell zusätzlich eingefügten Pfade waren von der Entscheidungssicherheit, den richtigen Betrieb gewählt zu haben am Ende des 9. Schuljahrs auf die Zufriedenheit mit dem Betrieb $(\beta=.12, p<.01)$ und auf die Absicht, die Lehre abschliessen zu wollen $(\beta=.14, p<.01)$ im sechsten Monat der beruflichen Grundbildung. Die wahrgenommene Passung mit dem Lehrberuf und die Entscheidungssicherheit, den richtigen Beruf und den richtigen Betrieb gewählt zu haben, sind wichtige Variablen in der Vorhersage der wahrgenommenen Passung mit dem Lehrberuf und der distalen Ergebnisse der betrieblichen Sozialisation (Abbildung 3).

In Abbildung 3 sind zusätzlich die statistisch signifikanten Korrelationen zwischen den Variablen im 9. Schuljahr, respektive den Variablen im sechsten Monat der beruflichen Grundbildung aufgeführt. Im 9. Schuljahr korrelierten die Zufriedenheit mit der beruflichen Grundbildung, die Zufriedenheit mit dem Betrieb und der Entscheidungssicherheit, den passenden Beruf und den passenden Lehrbetrieb gewählt zu haben, hoch miteinander. Am höchsten korrelierte die Entscheidungssicherheit, den richtigen Beruf und die Entscheidungssicherheit, den richtigen Lehrbetrieb gewählt zu haben, mit $r=.63, p<.01$. Am geringsten korrelierte die Entscheidungssicherheit, den richtigen Lehrbetrieb gewählt zu haben mit der Absicht, die Lehre abschliessen zu wollen mit $r=.26, p<.01$. Die Ergebnisse des Sozialisationsprozesses im sechsten Monat der Lehre waren ebenfalls korreliert. Für die beiden Zufriedenheitsmasse (Lehre, Betrieb) lag die Korrelation bei $r=.50, p<.01$, alle andern Korrelationen waren tiefer. 


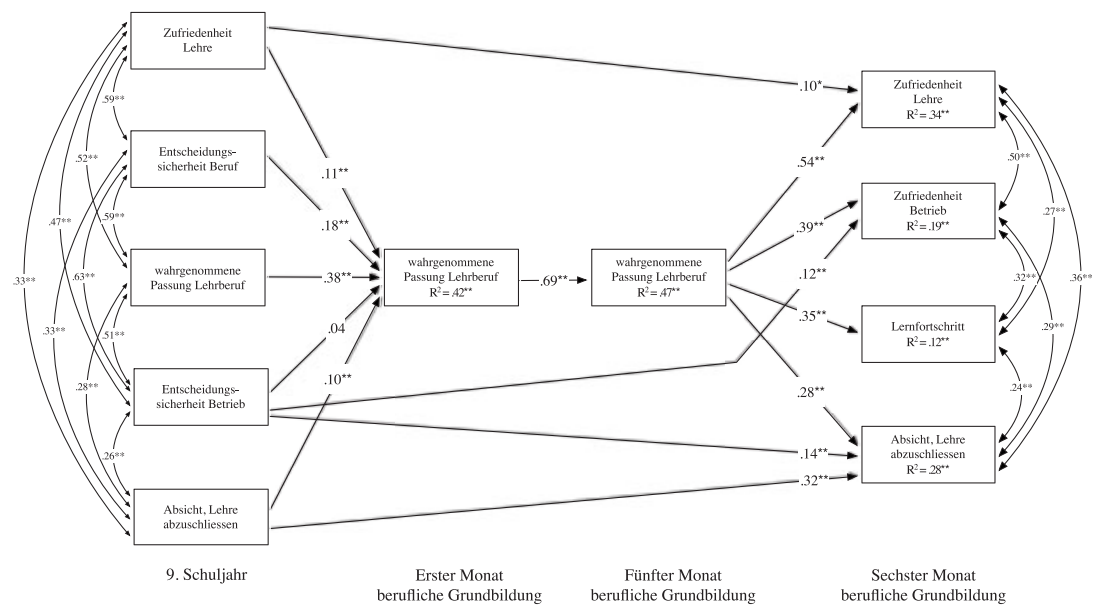

Abbildung 3 Endgültiges Modell, $\chi 2(25)=38.91, p<.05$, CFI $=.987$, TLI $=.977$, SRMR $=$ .065 , RMSEA $=.037, N=409$; Werte bei gerichteten Pfeilen sind standardisierte Pfadkoeffizienten; Linien mit Doppelpfeilen sind Korrelationen; ${ }^{* *}=$ $p<.01$, übrige Werte sind statistisch nicht signifikant.

\subsection{Diskussion}

Schülerinnen und Schüler mit einem direkten Einstieg in eine berufliche Grundbildung hatten am Ende des 9. Schuljahrs eine insgesamt hohe wahrgenommene Passung mit dem Lehrberuf. Mit Beginn der beruflichen Grundbildung stieg die wahrgenommene Passung mit dem Lehrberuf im Mittel leicht an und ging anschliessend bis zum fünften Monat leicht zurück. Es konnte bereits in einer früheren Studie gezeigt werden, dass sich bei erwartungskonformen Übergangen die wahrgenommene Passung nur wenig verändert (Neuenschwander, 2007). Die hier präsentierten Ergebnisse bestätigen dies. Dennoch gibt es Jugendliche, bei denen die wahrgenommene Passung mit dem Lehrberuf beim Übergang von der Schule in die berufliche Grundbildung sank (Abbildung 2). Sank die wahrgenommene Passung mit dem Lehrberuf beim Übergang von der Schule in die berufliche Grundbildung, bestand ein erhöhtes Risiko, dass die wahrgenommene Passung mit dem Lehrberuf bis in den fünften Monat weiter sank (bei 15\% der Jugendlichen) und auch nicht wieder stieg. Eine negative Entwicklung der wahrgenommen Passung mit dem Lehrberuf scheint sich zu verstetigen, auch wenn 
dies angesichts der kurzen Beobachtungszeit von sechs Monaten vorsichtig interpretiert werden muss.

Die Ergebnisse zeigen, dass die Jugendlichen am Ende des 9. Schuljahrs eine hohe wahrgenommene Passung mit dem Lehrberuf haben, sie sind sich in ihrer Entscheidung der Wahl der Lehre sicher und damit zufrieden. Sie haben zudem die Absicht, die Lehre abzuschliessen. Wir stellen fest, dass aufgrund dieser Faktoren die wahrgenommene Passung mit dem Lehrberuf im ersten Monat der beruflichen Grundbildung sehr gut vorhergesagt werden kann (43\% erklärte Varianz). Das heisst, dass die wahrgenommene Passung mit dem Lehrberuf im ersten Monat der beruflichen Grundbildung sehr gut mit der wahrgenommenen Passung mit dem Lehrberuf im 9. Schuljahr übereinstimmt.

Die Ergebnisse der Pfadanalyse zeigen weiter, dass die wahrgenommene Passung mit dem Lehrberuf im fünften Monat der beruflichen Grundbildung ein guter Indikator distaler Ergebnisse der beruflichen Sozialisation ist. Die wahrgenommene Passung mit dem Lehrberuf kann so als Indikator für eine erfolgreiche Anpassung verwendet werden. Die distalen Ergebnisse der beruflichen Sozialisation (die Zufriedenheit mit der Lehre, die Zufriedenheit mit dem Betrieb, der Lernfortschritt und die Absicht, die Lehre abzuschliessen) werden von der wahrgenommenen Passung im fünften Monat der beruflichen Grundbildung in unterschiedlichem Ausmass vorhergesagt. Den grössten Einfluss hat die wahrgenommene Passung auf die Zufriedenheit mit der Lehre, in etwas geringerem Ausmass auf die Zufriedenheit mit dem Betrieb und den Lernfortschritt und den geringsten Einfluss auf die Absicht, die Lehre abzuschliessen.

Unabhängig von der wahrgenommenen Passung mit dem Lehrberuf hatte die Absicht, die Lehre abzuschliessen im 9. Schuljahr, einen grossen Einfluss auf die Absicht, die Lehre abzuschliessen im sechsten Monat der berulichen Grundbildung. Die Absicht, die Lehre abzuschliessen im 9. Schuljahr, war ein eher schwacher Prädiktor der wahrgenommenen Passung mit dem Lehrberuf im ersten Monat der beruflichen Grundbildung. Dies lässt vermuten, dass die wahrgenommene Passung und die Absicht, die Lehre abzuschliessen, zwei aufeinander bezogene, aber doch auch unabhängige Prozesse beschreiben.

Die zusätzlich ins Modell aufgenommenen Pfade von der Entscheidungssicherheit, den richtigen Betrieb gewählt zu haben, auf die Zufriedenheit mit dem Betrieb und die Absicht, die Lehre abzuschliessen im sechsten Monat der beruflichen Grundbildung weisen darauf hin, dass die Wahl des Ausbildungsbetriebs unabhängig von der Wahl des Lehrberufs wichtig ist. Die Sicherheit in der Entscheidung für den Lehrbetrieb wirkt sich aber nicht unmittelbar im Übertritt von der Schule in die berufliche Grundbildung aus, sondern beginnt sich erst im Verlauf der ersten Monate der beruflichen Grundbildung zu zeigen. Dieses Ergebnis 
zeigt, dass neben der Suche nach dem richtigen Beruf die Frage nach dem richtigen Lehr- und Ausbildungsbetrieb mehr Beachtung finden sollte. Dies bestätigt Ergebnisse aus der Forschung zu Lehrabbrüchen, die als eine wichtige Ursache eine falsche Lehrbetriebswahl identifiziert hat (Stalder \& Schmid, 2012).

Insgesamt zeigen die Ergebnisse, dass die im Berufswahlprozess erarbeitete wahrgenommene Passung mit dem Lehrberuf und die Entscheidungssicherheit wichtige Faktoren sind, die zu einer hohen Passung zu Beginn der beruflichen Grundbildung beitragen. Während der ersten Monate der beruflichen Grundbildung ist die durchschnittliche Ausprägung der wahrgenommenen Passung relativ stabil, wobei Sie für eine Gruppe von Jugendlichen eher noch zunimmt, während sie für eine andere Gruppe von Jugendlichen kontinuierlich sinkt. Die wahrgenommene Passung mit dem Lehrberuf ist ein guter Prädiktor für die distalen Ergebnisse der betrieblichen Sozialisation. Die präsentierten Ergebnisse deuten darauf hin, dass es für den Übertritt von der Schule in die berufliche Grundbildung wichtig ist, eine hohe wahrgenommene Passung mit dem Lehrberuf zu haben, die auch auf einer Sicherheit in der Entscheidung für einen bestimmten Ausbildungsbetrieb beruht. Die wahrgenommene Passung ist überdies ein guter Indikator für betriebliche Anpassungsprozesse.

\subsection{Theoretische Implikationen}

Auch wenn sich die sozialen Situationen in Schule und Ausbildungsbetrieb stark voneinander unterscheiden, scheint der Übergang von der Schule in die berufliche Grundbildung für die Jugendlichen mit einem direkten Beginn der beruflichen Grundbildung in der Regel gut gemeistert zu werden, anders als dies in anderen Bildungssystemen zum Beispiel beim Übergang vom College zur Arbeit der Fall zu sein scheint (Wendlandt \& Rochlen, 2008). Entsprechend sollte in Theorien der beruflichen Sozialisation beim Eintritt in die berufliche Grundbildung das Ergebnis des Berufswahlprozesses hohes Gewicht erhalten. Jugendliche bereiten sich im Berufswahlprozess ausführlich und erfolgreich auf die neuen Anforderungen vor und scheinen dadurch gut vorbereitet zu sein. Die Anpassung an die neue Ausbildungs- und Arbeitssituation in den ersten Monaten sind wichtig (Bauer \& Erdogan, 2011), doch es ist noch wichtiger, dass sich die Jugendlichen in ihrer Entscheidung, den richtigen Lehrberuf und den richtigen Ausbildungsbetrieb gewählt zu haben, sicher sind. Der Entscheidungssicherheit sollte in weiteren Studien mehr Aufmerksamkeit zukommen. 


\subsection{Praktische Implikationen}

Der Berufswahlprozess darf sich nicht nur auf die Wahl des Lehrberufs und der passenden Lehre beschränken. Vielmehr sollte der Wahl des richtigen Lehrbetriebs mehr Aufmerksamkeit geschenkt werden, während des schulischen Berufswahlunterrichts und vor allem auch während der Schnupperlehren und dem Bewerbungs- und Selektionsprozess. Die Jugendlichen können den Ausbildungsbetrieb jedoch nur sehr eingeschränkt selber wählen. Sie entscheiden sich, ob sie sich für eine bestimmte Schnupperlehre oder Lehrstelle bewerben wollen oder nicht. Über ihre Bewerbung entscheidet dann aber der Ausbildungsbetrieb. Hier liegt eine grosse Verantwortung bei den Betrieben, die durch Schnupperlehren und die Gestaltung des Bewerbungs- und Selektionsprozesses dazu beitragen können, dass die Jugendlichen in den passenden Ausbildungsbetrieb aufgenommen werden. So erhalten Jugendliche in Schnupperlehren einen Einblick in die betriebliche Realität und gewinnen Entscheidungssicherheit (Neuenschwander \& Hermann, 2014), wenn diese gut vorbereitet, durchgeführt und nachbereitet werden. Betriebe und Jugendliche könnten Schnupperlehren oder Bewerbungsgespräche vermehrt nutzen, um den Jugendlichen eine grössere Sicherheit in ihrer Entscheidung zu ermöglichen. Zur Gestaltung und Wirkung von Schnupperlehren und den Selektionsprozessen liegen allerdings kaum relevante Studien vor (Imdorf, 2007; Neuenschwander, 2010; Stalder, 2000). Durch eine bessere Vorbereitung und Selektion kann früh erkannt werden, ob die zukünftigen Lernenden und die Ausbildungsbetriebe zueinander passen. Schnupperlehren sollten genutzt werden, um den Jugendlichen einen realistischen Blick auf den zukünftigen Lehrberuf zu ermöglichen, da dies die wahrgenommene Passung erhöht (Vandenberg \& Scarpello, 1990).

Die Jugendlichen, die direkt nach der Schule mit einer beruflichen Grundbildung beginnen, sind sich sicher, den richtigen Lehrberuf gewählt zu haben. Dies ändert sich im ersten Monat der beruflichen Grundbildung nur wenig. Bis zum fünften Monat gibt es dann aber trotz einer hohen Stabilität doch Veränderungen, die sich im fünften Monat der beruflichen Grundbildung in einer hohen wahrgenommenen Passung mit dem Lehrberuf zeigen. Jugendliche mit einer hohen wahrgenommenen Passung im fünften Monat, haben im sechsten Monat eine grössere Zufriedenheit mit der Lehre, eine grösser Zufriedenheit mit dem Betrieb, bewerten ihren Lernfortschritt besser und sind überzeugter davon, dass sie die Lehre abschliessen wollen. Wenn Schülerinnen und Schüler und Lernende über eine gering oder geringer werdende wahrgenommene Passung mit dem Lehrberuf berichten, ist dies ein Hinweis, dass eine negative Entwicklung einsetzt. Wenn eine sinkende wahrgenommen Passung erkannt wird, hilft dies frühzeitig, un- 
günstige Verläufe zu identifizieren. Es eröffnet die Möglichkeit, Gegenmassnahmen zu ergreifen.

\section{Literatur}

Abele-Brehm, A. E. (2014). The influence of career success on subjective well-being. In A. C. Keller, R. Samuel, M. M. Bergman, \& N. K. Semmer (Eds.), Psychological, educational, and sociological perspectives on success and well-being in career development (pp. 7-18). Dordrecht, NL: Springer Sience+Business Media

Ajzen, I. (2002). Perceived behavioral control, self-efficacy, locus of control, and the theory of planned behavior. Journal of Applied Social Psychology, 32, 665-683.

Bauer, T. N., \& Erdogan, B. (2011). Organizational socialization: The effective onboarding of new employees. In S. Zedeck (Ed.), APA Handbook of industrial and organizational psychology. Maintaining, expanding and contracting the organization (pp. 51-64). Washington, DC: American Psychological Association.

Bergmann, C. (2007). Berufliche Interessen und Berufswahl. In H. Schuler \& K. Sonntag (Hrsg.), Handbuch der Arbeits- und Organisationspsychologie (pp. 413-421). Göttingen, D: Hogrefe Verlag GmbH \& Co. KG.

Berweger, S., Krattenmacher, S., Salzmann, P., \& Schönenberger, S. (2013). LiSa. Lernende im Spannungsfeld von Ausbildungserwartungen, Ausbildungsrealität und erfolgreicher Erstausbildung. St. Gallen, CH: Pädagogische Hochschule St. Gallen, Insitut Professionsforschung und Kompetenzentwicklung.

Cable, D. M., \& Judge, T. A. (1996). Person-organization fit, job choice decisions, and organizational entry. Organizational Behavior and Human Decision Processes, 67, 294-311. doi: 10.1037/0021-9010.92.5.1446

Firth, L., Mellor, D. J., Moore, K. a., \& Loquet, C. (2004). How can managers reduce employee intention to quit? Journal of Managerial Psychology, 19, 170-187.

Görlich, Y., \& Schuler, H. (2007). Personalentscheidung und Nutzen. In H. Schuler \& K. Sonntag (Eds.), Handbuch der Arbeits- und Organisationspsychologie (S. 520-527). Göttingen, D: Hogrefe Verlag GmbH \& Co. KG.

Herzog, W., Neuenschwander, M. P., \& Wannack, E. (2001). Berufswahlprozess bei Jugendlichen. Bern, CH: Universität Bern, Institut für Pädagogik und Schulpädagogik, Abteilung Pädagogische Psychologie.

Herzog, W., Neuenschwander, M. P., \& Wannack, E. (2006). Berufswahlprozess: Wie sich Jugendliche auf ihren Beruf vorbereiten. Bern, CH: Haupt.

Hirschi, A. (2013). Berufswahltheorien - Entwicklung und Stand der Diskussion. In T. Brüggemann \& S. Rahn (Eds.), Berufsorientierung. Ein Lehr- und Arbeitsbuch (S. 27-41). Münster, D: Waxmann.

Hirschi, A., Niles, S. G., \& Akos, P. (2011). Engagement in adolescent career preparation: social support, personality and the development of choice decidedness and congruence. Journal of Adolescence, 34, 173-182.

Holland, J. L. (1959). A theory of vocational choice. Journal of Counseling Psychology, 6(1), 35-45. 
Hu, L.-t., \& Bentler, P. M. (1999). Cutoff criteria for fit indexes in covariance structure analysis: Conventional criteria versus new alternatives. Structural Equation Modeling: A Multidisciplinary Journal, 6(1), 1-55. doi: 10.1080/10705519909540118

Imdorf, C. (2007). Lehrlingsselektion in KMU. Freiburg, CH: Heilpädagogisches Institut der Universität Freiburg.

Jones, G. R. (1986). Socialization tactics, self-efficacy, and newcomers' adjustments to organizations. Academy of Management Journal, 29, 262-279.

Kammeyer-Mueller, J. D., \& Wanberg, C. R. (2003). Unwrapping the organizational entry process: Disentangling multiple antecedents and their pathways to adjustment. Journal of Applied Psychology, 88(5), 779-794. doi: 10.1037/0021-9010.88.5.779

Kammeyer-Mueller, J. D., Wanberg, C. R., Rubenstein, A., \& Song, Z. (2013). Support, undermining, and newcomer socialization: Fitting in during the first 90 days. Academy of Management Journal, 56(4), 1104-1124. doi: 10.5465/amj.2010.0791

Keller, A. C., Semmer, N. K., Samuel, R., \& Bergman, M. M. (2014). The meaning and measurement of well-being as an indicator of success. In A. C. Keller, R. Samuel, M. M. Bergman, \& N. K. Semmer (Eds.), Psychological, educational, and sociological perspectives on success and well-being in career development (pp. 171-193). Dordrecht, NL: Springer Sience+Business Media

Keller, A. C., \& Stalder, B. E. (2012). Fluktuationsabsichten junger Erwachsener aus psychologischer Sicht: Die Rolle von Commitment und Laufbahnzufriedenheit. In M. M. Bergman, S. Hupka-Brunner, T. Meyer, \& R. Samuel (Hrsg.), Bildung - Arbeit - Erwachsenwerden. Ein interdisziplinärer Blick auf die Transition im Jugend- und jungen Erwachsenenalter (S. 95-112). Wiesbaden, D: Springer Fachmedien.

Krauth, J. (1993). Einführung in die Konfigurationsfrequenzanalyse (KFA). Ein multivariates nichtparamerisches Verfahren zum Nachweis und zur Interpretation von Typen und Syndromen. Weinheim, D: Beltz. Psychologie-Verlags-Union.

Kristof, A. L. (1996). Person-organization fit: An integrative review of its conceptualizations, measurement, and implications. Personnel Psychology, 49, 1-49.

Kristof-Brown, A. L., Zimmerman, R. D., \& Johnson, E. C. (2005). Consequences of individuals' fit at work: A meta-analysis of person-job, person-organization, persongroup, and person-supervisor fit. Personnel Psychology, 58, 281-342. doi: 10.1111/j.17446570.2005.00672.x

Lengnick-Hall, M. L., Lengnick-Hall, C. A., Andrade, L. S., \& Drake, B. (2009). Strategic human resource management: The evolution of the field. Human Resource Management Review, 19(2), 64-85. doi: 10.1016/j.hrmr.2009.01.002

Muthén, L. K., \& Muthén, B. O. (1998-2012). Mplus user's guide. Seventh edition. Los Angeles, CA: Muthén \& Muthén.

Nägele, C. (2013). Correlates and predictors of apprentices' perception of their workplace as learning place. An analysis of the first three years in apprenticeship. In S. Akoojee, P. Gonon, U. Hauschildt, \& C. Hofmann (Eds.), Apprenticeship in a globalised world. Premises, promises and pitfalls (Vol. 27, pp. 95-98). Münster, D: Lit.

Nägele, C., \& Neuenschwander, M. P. (2014). Adjustment processes and fit perceptions as predictors of organizational commitment and occupational commitment of young workers. Journal of Vocational Behavior, 85(3), 385-393. doi: dx.doi.org/10.1016/j. jvb.2014.08.011

Neuenschwander, M. P. (1997). Lehrvertragsauflösungen und ihre Folgen. Panorama, 4, 27-28. 
Neuenschwander, M. P. (2007). Bedingungen und Anpassungsprozesse bei erwartungswidrigen Bildungsverläufen. In T. Eckert (Hrsg.), Übergänge im Bildungswesen (pp. 83104). Münster, D: Waxmann Verlag.

Neuenschwander, M. P. (2010). Selektionsprozesse beim Übergang von der Primarschule in die Berufsbildung. Schulübergang und Selektion, 15-34.

Neuenschwander, M. P. (2011). Determinanten der Passungswahrnehmung nach dem Übergang in die Sekundarstufe II. Schweizerische Zeitschrift für Bildungswissenschaften, 33, 401-420.

Neuenschwander, M. P., \& Frank, N. (2009). Familie-Schule-Beruf FASE B. Dokumentation der Schülerbefragung Erhebung 2008. Solothurn, CH: Fachhochschule Nordwestschweiz, Pädagogische Hochschule, Institut Forschung und Entwicklung, Zentrum Lernen und Sozialisation.

Neuenschwander, M. P., Gerber, M., Frank, N., \& Bosshard, S. (2013). (t6 L) Sozialisationsprozesse beim Übergang in den Lehrbetrieb (SoLe). Dokumentation der Lernendenbefragung. Schlusserhebung. Solothurn, CH: Fachhochschule Nordwestschweiz, Pädagogische Hochschule, Institut Forschung und Entwicklung, Zentrum Lernen und Sozialisation.

Neuenschwander, M. P., Gerber, M., Frank, N., \& Rottermann, B. (2012). Schule und Beruf: Wege in die Erwerbstätigkeit. Wiesbaden, D: VS Verlag für Sozialwissenschaften.

Neuenschwander, M. P., \& Hermann, M. (2014). Determinanten von Berufsbildungsentscheidungen bei eingeschränkter Informationsbasis. In A. B. Liegmann, I. Mammes, \& K. Racherbäumer (Hrsg.), Facetten von Übergängen im Bildungssystem - Nationale und internationale Ergebnisse empirischer Forschung (pp. 125-140). Münster, D: Waxmann.

Neuenschwander, M. P., \& Nägele, C. (2014). Sozialisationsprozesse beim Übergang in den Lehrbetrieb (SoLe). Schlussbericht im Auftrag des SBFI. Solothurn, CH: Fachhochschule Nordwestschweiz, Pädagogische Hochschule, Institut Forschung und Entwicklung, Zentrum Lernen und Sozialisation.

Schmid, E. (2010). Kritisches Lebensereignis «Lehrvertragsauflösung». Eine Längsschnittuntersuchung zum Wiedereinstieg und zum subjektiven Wohlbefinden betroffener Jugendlicher. Bern, $\mathrm{CH}$ : hep-Verlag.

Schumacker, R. E., \& Lomax, R. G. (1996). A beginner's guide to structural equation modeling. Mahwah, NJ: Lawrence Erlbaum Associates, Inc. .

Singer, A., Gerber, M., \& Neuenschwander, M. P. (2014). Individuelle und soziale Bedingungen der beruflichen Sozialisation im Lehrbetrieb. In M. P. Neuenschwander (Hrsg.), Selektion in Schule und Arbeitsmarkt (pp. 165-186). Zürich, CH: Rüegger.

Stalder, B. E. (2000). Gesucht wird... Rekrutierung und Selektion von Lehrlingen im Kanton Bern. Bern, CH: Amt für Bildungsforschung, Erziehungsdirektion des Kantons Bern.

Stalder, B. E., \& Schmid, E. (2012). Zurück zum Start? Berufswahlprozesse und Ausbildungserfolg nach Lehrvertragsauflösungen. In M. M. Bergman, S. Hupka-Brunner, T. Meyer, \& R. Samuel (Hrsg.), Bildung - Arbeit - Erwachsenwerden. Ein interdisziplinärer Blick auf die Transition im Jugend und jungen Erwachsenenalter (S. 265-285). Wiesbaden, D: Springer VS.

Tabachnick, B. G., \& Fidell, L. S. (2007). Using multivariate statistics. Boston, MA: Pearson/ Allyn \& Bacon. 
Taris, T. W., Feij, J. A., \& Capel, S. (2006). Great expectations - and what comes of it: The effects of unmet expectations on work motivation and outcomes among newcomers. International Journal of Selection and Assessment, 14, 256-268.

Vandenberg, R. J., \& Scarpello, V. (1990). The matching model: An examination of the processes underlying realistic job previews. Journal of Applied Psychology, 75(1), 60-67.

Vogel, R. M., \& Feldman, D. C. (2009). Integrating the levels of person-environment fit: The roles of vocational fit and group fit. Journal of Vocational Behavior, 75(1), 68-81. doi: 10.1016/j.jvb.2009.03.007

von Eye, A. (2002). Configural frequency analysis. Methods, models, and applications. Mahwah, NJ: Lawrence Erlbaum Associates, Publishers.

Wendlandt, N. M., \& Rochlen, A. B. (2008). Addressing the college-to-work transition: Implications for university career counselors. Journal of Career Development, 35(2), 151165. doi: dx.doi.org/10.1177/0894845308325646

Werbel, J., Landau, J., \& DeCarlo, T. E. (1996). The relationship of pre-entry variables to early employment organizational commitment. Journal of Personal Selling \& Sales Management, XVI(2), 25-36.

Dieses Buch wird unter der Creative Commons Namensnennung-Nicht kommerziell 4.0 International Lizenz (http://creativecommons.org/licenses/by-nc/4.0/deed.de) veröffentlicht, welche für nicht kommerzielle Zwecke die Nutzung, Vervielfältigung, Bearbeitung, Verbreitung und Wiedergabe in jeglichem Medium und Format erlaubt, sofern Sie den/die ursprünglichen Autor(en) und die Quelle ordnungsgemäß nennen, einen Link zur Creative Commons Lizenz beifügen und angegeben, ob Änderungen vorgenommen wurden. 